\title{
Studying Lorentz-odd CPT-even electrodynamics with waveguides
}

\author{
Davidson R. Viana* \\ Universidade Federal de Viçosa \\ Viçosa, Brazil \\ E-mail: davidsonvianadyahoo.com.br
}

\section{André H. Gomes, Jakson M. Fonseca, Winder A. Moura-Melo}

Universidade Federal de Viçosa

Viçosa, Brazil

E-mail: winderdufv.br

\begin{abstract}
We study a modified classical electromagnetism in $(3+1)$ dimensions, whose lagrangian includes a term proportional to a (constant) background tensorial object that breaks the Lorentz symmetry (active Lorentz or particle transformation), but respects CPT. Our attention is devoted to the plane wave-like solutions in a particular submodel. We study possible modes of propagation inside two different enviroments: a rectangular waveguide and coaxial transmission line.
\end{abstract}

4th International Conference on Fundamental Interactions -ICFI2010,

August 1-7, 2010

Viçosa, Brazil

\footnotetext{
* Speaker.
} 


\section{Introduction and Motivation}

Planck energy scale is still far beyond current experimental possibilities. Invariance under Lorentz and CPT transformations is a fundamental requirement of local relativistic quantum field theories, including the Standard Model (SM) of particle physics. A question of interest is whether the Lorentz symmetry remains valid at these extremely high energies, whose answer certainly would be useful whenever building grand unified theories, in which general relativity were consistently accommodated. Here, we study a reminiscent of this possible symmetry violation, incorporated in the body of the so-called Standard Model Extension (SME). The radiation sector from the SME, which incorporates in its Lagrangian terms with vector and / or background tensor fields, has been studied, including several experimental data. There is a great deal of efforts to detect parameters associated with background tensor and vector fields and to set upper bounds

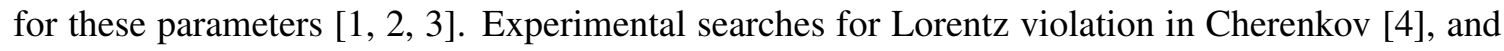
synchrotron [5] radiations, cosmic microwave background [四, among many others, have seen reported. Theoretical proposals have emerged to investigate ways to measure parameters of violation of the SME through the confinement of electromagnetic waves, for example, in resonant cavities and waveguides $[\because, \square, 8]$ the latter being the subject of interest in this work. We present some properties of electrodynamic model that violate Lorentz symmetry but preserves CPT. We analyse such a model in two types of waveguides, which rectangular cross section and the so called coaxial transmission line and rectangular waveguide, which exhibit distinct modes of propagation of electromagnetic waves. The parameters associated to the Lorentz breaking, considering our proposed solution, alter the properties of the radiation within the guide, which is evidenced in the equations of modified electrodynamics to describe the dynamics of waves.

\section{The Model and its basic features}

The SM of elementary particles describes all fundamental particles and their interactions (except gravity). His extension proposed in recent decades, the SME, shares the same properties of the SM (such as conservation of energy and momentum, microcausality, renormalizability by power counting and others), except that the symmetries of Lorentz and $C P T$ can be violated [Q]. In this work, our attention is devoted to radiation sector (or pure-photon sector) of the SME, whose lagrangian has two terms beyond the Maxwell's model (without sources), namely:

$$
\mathscr{L}_{\text {total }}=-\frac{1}{4} F_{\mu v} F^{\mu v}-\frac{1}{4}\left(k_{F}\right)_{\kappa \lambda \mu v} F^{\kappa \lambda} F^{\mu v}
$$

The tensorial object of rank $4,\left(k_{F}\right)_{\kappa \lambda \mu \nu}$, has no dimension and is assumed as a set of constants coupling the electromagnetic tensor to itself (a kind of correction to the usual kinetic term, $\left(\frac{1}{4} F_{\mu v} F^{\mu v}\right)$. This object has the symmetries of the Riemann's curvature tensor,

$$
\left(k_{F}\right)^{\mu v \alpha \beta}=\left(k_{F}\right)^{\alpha \beta \mu \nu}=-\left(k_{F}\right)^{v \mu \alpha \beta}=\left(k_{F}\right)^{v \mu \beta \alpha},
$$

the Bianchi identity for rank 4 tensors: $\left(k_{F}\right)^{\mu v \alpha \beta}+\left(k_{F}\right)^{\mu \beta v \alpha}+\left(k_{F}\right)^{\mu \alpha \beta v}=0$, and also zero double trace, $\left(k_{F}\right)_{\mu \nu}{ }^{\mu v}=0$, so that the 256 components that constitute only 19 are linearly independent. Experimental tests require more precise limits on the size of the parameters, and the best 
estimates have been obtained from measurements of cosmological birefringence of light, so that $\left|\left(k_{A F}\right)^{\mu v \alpha \beta}\right| \leqslant 10^{-32}[\mathbb{[}]$ ]. That is, the violations described herein would be extremely small and therefore, difficult to be detected, which on the other hand, gives them some possibility of real existence []]. The motion equation derived from the lagrangian,

$$
\partial_{\alpha} F_{\mu}^{\alpha}+\left(k_{F}\right)_{\mu \alpha \beta \gamma} \partial^{\alpha} F^{\beta \gamma}=0
$$

and the Bianchi identity,

$$
\partial_{\mu} \widetilde{F}^{\mu v}=0
$$

form a set of linear equations in the tensor $F^{\mu \nu}$ and potential $A^{\mu}$, which give us the modified Maxwell's equations where the discrete symmetry $C P T$ is preserved.

Our attention will be devoted to a submodel which we setting a component of tensor $\left(k_{F}\right)$ odd under parity (and under time reversal) is one of which lead to birefringence: $\left(k_{F}\right)^{0123}$. By analyzing all those that relate to this through the symmetries of the tensor background, we obtain a set of equations that relate its components. In particular we choose only the case where $\left(k_{F}\right)^{\mu v \alpha \beta}$ has index $\mu, v, \alpha, \beta$ completely different. Assuming it follows the analysis of some parameters that couple the electric and magnetic field in the lagrangian (2.]), where there is an explicit coupling in $\vec{E}$ and $\vec{B}$. This submodel, which is the main focus of this work, includes corrections depending on the direction we take to the electromagnetic waves, as we shall see later in this article. In this submodel we have three components, two of which are related by the symmetry of Riemann tensor and the Bianchi identity for tensors of rank 4 as in the following equation:

$$
\left(k_{F}\right)^{0123}+\left(k_{F}\right)^{0312}+\left(k_{F}\right)^{0231}=0 .
$$

Naming $\left(k_{F}\right)^{0123}=\xi,\left(k_{F}\right)^{0312}=\rho$ e $\left(k_{F}\right)^{0231}=\sigma$. It is convenient to use these coefficients to write the modified Maxwell's equations in vacuum as 3-vector formalism in cartesian coordinates:

$$
\begin{aligned}
\vec{\nabla} \cdot \vec{E}= & 2 \xi \partial_{x} B_{x}-2 \rho \partial_{z} B_{z}-2 \sigma \partial_{y} B_{y} \\
\vec{\nabla} \times \vec{B}-\partial_{t} \vec{E}= & \left(2 \xi \partial_{t} B_{x}+2 \rho \partial_{y} E_{z}-2 \sigma \partial_{z} E_{y}\right) \hat{x}+ \\
& +\left(2 \xi \partial_{z} E_{x}-2 \rho \partial_{x} E_{z}+2 \sigma \partial_{t} B_{y}\right) \hat{y}+ \\
& +\left(-2 \xi \partial_{y} E_{x}+2 \rho \partial_{t} B_{z}+2 \sigma \partial_{x} E_{y}\right) \hat{z}
\end{aligned}
$$

All equations have linear corrections in the parameters associated to Lorentz breaking, even in the static limit. The equations coming from the Bianchi identity remain unchanged. The fields appear as sources for themselves with new derivative that appear on the right side.

\section{Modified electrodynamics in a coaxial transmission line}

In this section we studied the confinement that occurs inside a coaxial transmission line. This consists of a long straight conducting cylinder of radius $a$ surrounded by a hollow cylindrical surface also conductive, with constant radius $b$ and also constant cross section, and $b>a$. In the coaxial cable, it is possible the propagation of electromagnetic waves in transverse electromagnetic 
modes (TEM), characterized by the nullity of components of electric and magnetic fields in the direction of the waveguide, or $E_{z}=B_{z}=0$. [12, [12]].

For the equations $\sqrt{2.6}$ and $\sqrt{2.7}$ we supposed solutions like:

$$
\begin{aligned}
\vec{E}(x, y, z, t) & =\vec{E}_{0}(x, y) e^{i(k z-\omega t)} \\
\vec{B}(x, y, z, t) & =\vec{B}_{0}(x, y) e^{i(k z-\omega t)}
\end{aligned}
$$

Setting $E_{z}=B_{z}=0$ and replacing (B.D) e (B.2) in modified Maxwell's equations, we obtained the corrections associated to frequency (or the dispersion relation), $\omega=|\vec{k}|(1 \pm|\xi-\sigma|)$, and through this, considering only leading order corrections, we calculate the group and phase velocities (coincidentally, the same), $v_{\text {group }}=v_{\text {phase }}=c(1 \pm|\xi-\sigma|)$. There are two values for the velocities (two modes of propagation), one of which is supraluminal. The parameter $\rho$ does not appear explicitly in the corrections. This fact makes no charge for this parameter, which in principle can be null or not according to the symmetry (2.5) that shows the dependency between the three parameters, $\xi+\rho+\sigma=0$. For simplicity, we assume solutions of the equations ([2.6)-(‥7) as the solutions of the equations of electrostatics and magnetostatics in cylindrical coordinates, with corrections in the frequency. After an expansion in power series, considering only the dominant corrections, the real part of each field takes the form:

$$
\begin{gathered}
\vec{E}=\frac{A_{0} \cos \left(k z-\omega^{\prime} t\right) \hat{s}}{s} \pm \frac{|\xi-\sigma| A_{0} \omega^{\prime} t \sin \left(k z-\omega^{\prime} t\right) \hat{s}}{s}+\mathscr{O}\left(|\xi-\sigma|^{2}\right) \\
\vec{B}=\frac{A_{0} \cos \left(k z-\omega^{\prime} t\right) \hat{\phi}}{c s} \pm \frac{|\xi-\sigma| A_{0} \omega^{\prime} t \sin \left(k z-\omega^{\prime} t\right) \hat{\phi}}{c s}+\mathscr{O}\left(|\xi-\sigma|^{2}\right),
\end{gathered}
$$

where $\omega^{\prime}=|\vec{k}|$ the frequency that is obtained in the usual electrodynamics. Using the equations (B.3) and (B.4) we obtain the time-averaged Poynting vector and the total radiated power with dominant corrections:

$$
\langle\vec{S}\rangle=\frac{A^{2}}{2 c s^{2}}[1 \mp|\xi-\sigma| \cos (2 k z)] \hat{z}+\mathscr{O}\left(|\xi-\sigma|^{2}\right) .
$$

The radiated power (time average) calculated using the Poynting vector is:

$$
\langle P\rangle=\frac{\pi A^{2}}{c} \ln \left(\frac{b}{a}\right)[1 \mp|\xi-\sigma| \cos (2 k z)]+\mathscr{O}\left(|\xi-\sigma|^{2}\right) .
$$

We see that these quantities have their values increased or decreased by small corrections linear in the parameters. Paying attention to the radial coordinate, $s$, we see that we can get more disparate values of the usual electrodynamics if the waveguide radius is extremely small. This is due to the fact that the parameters associated with the breaking of Lorentz hitherto not been detected and obtained more reliable values of cosmological measurements impose limits on the order of $10^{-32}$ [ए0]. For example, a guide with radius $s \sim 10^{-8} \mathrm{~m}$ would lead to corrections in irradiance by a factor of $10^{16} \times[|\xi-\sigma| \cos (k z)]$. The contribution associated with the breaking of Lorentz is evidenced also by the oscillation of the values of both the irradiance and power in the direction of the guide, since the term $\propto \cos (k z)$ does not appear in the usual results. Such differences allow to set limits for experimental parameters of breaking, or detect them. 


\section{Modified electrodynamics in a rectangular waveguide}

In a rectangular waveguide, the electromagnetic waves propagate in the cavity of the waveguide. We assume infinite length ( $z$ direction) so there are no edge effects. We assume that the walls of the guide are perfectly conducting (infinite conductivity), so that the electric field parallel to the wall vanishes, and the magnetic field perpendicular to these also vanishes, which formally follows from the boundary conditions, which are a consequence of Maxwell's equations homogeneous, unchanged for the breaking of Lorentz symmetry. As written: $\vec{E} \|=\overrightarrow{0}, B^{\perp}=0$. The boundaries of the guide (in Cartesian coordinates) are: $x=0, x=a$ e $y=0, y=b$. It is reasonable to assume general forms for the fields in the rectangular guide, for waves in the direction $z\left(k=k_{z}\right)$. For simplicity we take as proposed solution for each field, the "ansatz" [四]:

$$
\begin{aligned}
& \vec{E}=\left(E_{x} \hat{x}+E_{y} \hat{y}+E_{z} \hat{z}\right) e^{i(k z-\omega t)} \\
& \vec{B}=\left(B_{x} \hat{x}+B_{y} \hat{y}+B_{z} \hat{z}\right) e^{i(k z-\omega t)}
\end{aligned}
$$

this proposal associate corrections with the transverse components of the amplitudes which contains the fields transverse to the direction of motion and axial components. Corrections should contribute via relation between $\omega$ and $k$. Here we see that the dominant corrections are all linear. Replacing the proposed solution in the modified Maxwell's equations we obtain the differential equations for the axial components, which determine the dynamics of the fields:

$$
\begin{aligned}
& \left(\partial_{x}^{2}+\partial_{y}^{2}+\omega^{2}-k^{2}\right) E_{z}+\left(\frac{2 \sigma k^{2}-2 \xi k^{2}}{\omega^{2}-k^{2}}+2 \sigma\right) \partial_{x}^{2} B_{z}+\left(\frac{2 \xi k^{2}-2 \sigma k^{2}}{\omega^{2}-k^{2}}+2 \xi\right) \partial_{y}^{2} B_{z}+ \\
& +\frac{4 \xi \omega k}{\omega^{2}-k^{2}} \partial_{x} \partial_{y} E_{z}+2 \rho\left(\omega^{2}-k^{2}\right) B_{z}=0 \\
& \left(\partial_{x}^{2}+\partial_{y}^{2}+\omega^{2}-k^{2}\right) B_{z}+\left(\frac{2 \sigma \omega^{2}+2 \xi k^{2}}{\omega^{2}+k^{2}}-2 \rho\right) \partial_{x}^{2} E_{z}+\left(\frac{2 \xi \omega^{2}-2 \sigma k^{2}}{\omega^{2}-k^{2}}-2 \rho\right) \partial_{y}^{2} E_{z}+ \\
& +\frac{4(\sigma-\xi) \omega k}{\omega^{2}-k^{2}} \partial_{x} \partial_{y} B_{z}=0 .
\end{aligned}
$$

Unlike the usual case, we have a coupled system of linear differential equations of second order. These equations recover the usual case when the three parameters vanish. However, one can not say that they should be zero simultaneously. Until now we could choose any sub-model with $\xi=0$, or $\rho=0$, or $\sigma=0$, respecting the symmetry of the equation ([D.J), which prohibits the three parameters have the same sign or nullify two of them. The solutions (if any) of (4.3) and (4.4), which satisfy the boundary conditions of the waveguide, are not easy to be obtained analytically and numerically neither, since the parameters are very small and would be difficult to analyze the differences between these results and the usual case, for example, graphically, which justifies the analytical approach in the search for electric and magnetic transverse modes, which have simpler equations. 


\subsection{Transverse magnetic modes}

Transverse magnetic (TM) modes are obtained by making $B_{z}=0$. Initially, the usual case is recovered with the three parameters being null. Through a solution in the form of plane wave (4.3]), we obtained a dispersion relation with a small correction as regards the result known in the usual electrodynamics (where the parameters vanish), particularly only $\xi=0$ already covers the usual case. This same equation leads to the usual cutoff frequency [12], [3]]. However, the equation (4.4) leads to the zero cutoff frequency. The group and phase velocities obtained through (4.4) not recover the usual case and not presents small corrections, but lead to macroscopic values distinct of the usuals ones. In this way each equation arising from (4.3) and (4.4) implies different physical consequences. Assuming non nullity of these parameters there are strange values of physical quantities (such as phase and group velocities), not recovering the usual case.

\subsection{Transverse electric modes}

We obtain the transverse electric (TE) modes by setting $E_{z}=0$. Thus, we have two differential equations for the axial magnetic component, which lead to results that do not agree with the usual electrodynamics. In these modes there is a zero cutoff frequency such as in the TE mode in one of the equations. Therefore, by assuming the parameters $\rho, \xi$ and $\sigma$ are not null, we calculated the phase velocities and group velocities. These velocities are different from usual calculated by equation (4.3). Through (4.4) we have only very small corrections.

\section{Conclusions and perspectives}

Inside a coaxial transmission line, our findings indicate that Lorentz-breaking implies in small modifications of these usual results, perhaps beyond current experimental detection. In a waveguide of rectangular shape, the partial differential equations which describe the axial components of the fields in the waveguide are quite different from the usual and a dynamic modified of each mode are very complicated and the results show that physical quantities have been drastically altered since the parameters are assumed to be non-null. However there is motivation to study the rigorous validity of the plane-wave solution for this submodel and understand the reason for these discrepancies.

\section{Acknowledgments}

The author D. R. Viana is grateful to R. S. Ferreira for help with LaTeX. The authors thank to CAPES, CNPq and FAPEMIG for financial support.

\section{References}

[1] V. A. Kosteleký and M. Mewes, "Lorentz Violating Electrodynamics and the Cosmic Microwave Background", Phys. Rev. Lett. 99, 011601 (2007).

[2] V. A. Kostelecký and M. Mewes, "Cosmological Constraints on Lorentz Violation in Electrodynamics", arXiv:hep-ph/0111026v1 2 Nov. (2001). 
[3] V. A. Kostelecký and N. Russell, "Data Tables for Lorentz and CPT Violation", arXiv:0801.0287v1 [hep-ph] 1 Jan. (2008).

[4] B. Altschul, "Vacuum Cherenkov Radiation in Lorentz-Violating Theories Without CPT Violation" Phys Rev. Lett.98, 041603 (2007).

[5] B. Alstchul, "Lorentz Violation and Sincrotron Radiation", Phys Rev. D 72085003 (2005).

[6] M. Mewes and A. Petrof, "Cavity Tests of Parity-Odd Lorentz Violations in Electrodynamics", Phys. Rev. D 75, 056002 (2007).

[7] M. E. Tobar et al.,"New Methods of Testing Lorentz Violation in Electrodynamics", Phys. Rev. D 71, 025004 (2005).

[8] A. H. Gomes, J. M. Fonseca, W. A. Moura-Melo, A. R. Pereira, "Testing CPT- and Lorentz-odd electrodynamics with waveguides", Journal of High Energy Physics Number 5, 1-12, DOI: 10.1007/JHEP05(2010)104.

[9] D. Colladay and V. A. Kostelecký, "CPT Violation and the Standard Model", Phys. Rev. D 556760 (1997).

[10] R. Casana, M. M. Ferreira Jr, and C. E. H. Santos, "Classical solutions for the Lorentz-violating and CPT-even term of the standard model extension", Phys. Rev. D 78, 105014 (2008).

[11] F. R. Klinkhammer "New Indirect Bounds on Lorentz Violation in the Photon Sector", arXiv:0810.1446v3 [hep-ph] 27 Oct. (2008).

[12] J. D. Jackson, "Classical Electrodynamics", Wiley, New York, Third Edition (1999).

[13] D. J. Griffiths, "Introduction to Electrodynamics", Prentice Hall, Third Edition, (1999). 